\title{
ANALYSIS OF CHRONIC BURN DEPTH ASSESSMENT SYSTEM IN MULTIDIMENSIONAL IMAGE BASED ON TESTING RECORDS
}

\author{
Somashekhar G. C. \\ Professor, Electronics \& Communication Engg, Rajiv Gandhi Institute of Technology, \\ Bangalore, Karnataka, India \\ gcsomu@gmail.com \\ H. B. Phaniraju \\ Professor, Electrical \& Electronics Engg, Sri Devi Institute of Technology, \\ Tumkur, Karnataka, India
}

\begin{abstract}
The Recent Developments carried out in the bio- medical image processing plays a vital role in the assessment of the image processing systems. The research contributions carried out in this image processing field helps in assessing the images of burns using the machine learning algorithms and this is possible with the aid of Support vector-based Machine learning approaches. In this work, the focus is on the analysis of the chronic burnt images, assessing the depth of these images using multidimensional image. The methodologies involved in the work are Two stage SVM proposed as Enhanced Support Vector Machine (ESVM) which is of two stages, firstly the training stage and secondly, the classification stage.

The proposed ESVM is compared with supervised machine Learning (ML) algorithm with the precision, accuracy, success rate etc., and the ESVM out performs by $96.5 \%$ accuracy and $81.20 \%$ success rate 84.66\% Precision when compared with the existing algorithms and same is tested and validated in the study carried out and presented.
\end{abstract}

Keywords: Burnt Images; Image Processing; Machine Learning.

\section{Introduction}

Wound is defined as degradation or an injury in regular anatomy and as a function of patient's skin. These wounds begin to emerge from various pathological operations either externally or internally in any human organ. Wounds can be classified into Acute Wounds (AWs) and Chronic Wounds (CWs) based on its healing time. Acute wounds occur due to loss of a skin tissue, during a surgery or any unpredictable event or fall such as an accident. These wounds have the capacity of self/ natural healing within a short period of time in a systematic manner. While chronic wounds are exactly the opposite of acute wounds and do not have a property of selfhealing and takes longer than predicted for healing. Most common types of CWs are pressure ulcers (PUs), venous and arterial ulcers (VAUs), diabetic foot ulcers (DFUs) and burns, [Acha et al. (2013), Khalil et al. (2019), Wang et al. (2017)]. In this paper, we will deal with the diagnosis and treatment of chronic wounds, especially about burns. In order to analyze the CWs, we first have to recognize the area of the wound with respect to its various tissue types. During healing, we tend to have changes in the area of the wound. In order to make an estimate of the rate of healing, a trusted clinical technique monitored by a dermatologist is used. This adopted method is time consuming and may have errors.

Now establishing this fact with respect to burns, it is necessary that proper first aid is required for a burn injury. In order to know its exact source and healing possibilities, it is necessary to have information about the depth of the burn, and also depends upon specialized dermatological expertise. The cost of treating burns is very high and due to this we aim to have an automatic system at health care centers, where there is lack of specialists.

To overcome all of this backwardness, there came up a cost effective and quantitative solution that is a system which could segment and classify different wound tissues and provide an accurate assessment for different types of CWs, [Zhao et al. (2019), Dahel et al. (2003), Yadav et al. (2019)] [4][5][6]. These systems were called as Computer-Aided Diagnosis (CAD) system. For an instance, let's consider that a burn unit occupies a large 
geographic area where a patient is treated by a specialized doctor. The determination of burn depth and the possibility of the healing within 20 days is $52 \%$ as reported by less experienced surgeons. As per the experimental analysis the clinical estimate is between $65-75 \%$ of the final response. This justifies, why CAD tools acquired a greater importance in this field rather than the traditional methods.

Earlier we established the way in which CWs were used. The four different tissue types in CWs are necrotic eschar, slough, granulation and healing epithelial tissue. The healing process is recognized by the color changes from necrotic eschar to slough and to granulation in the wound, with this contest, it is clear that the wound, at its beginning stage will be comprising of the necrotic eschar and slough tissues. As the healing process begins, the granulation tissue begins to grow and this replaces the black yellow colored tissues. These tissues fill the wound cavity after which the healing tissue grows from the edges of the wound to color the area.

Burns are classified into - first degree burns, superficial dermal burns, deep dermal burns and full-thickness burns, according to their depth, [Georgopoulos et al. (2019)]. First degree burns, or epidermal burns are usually red, dry and painful and are not considered in order to assess the percentage of burnt are and the treatment process as they heal spontaneously. According to a plastic surgeon, burns are classifies as those that heal spontaneously and those that require excision. A false assessment of a burn might lead a patient to face needless surgery, hypertrophic scar formation, increased length of stay and risks contracture. Similar to epidermal burns, superficial burns are moist, red and painful to air and temperature, but do not need any clinical treatment as such, [Dai et al. (2020)]. The deep dermal burns and full thickness burns which are generally wet, waxy and leathery in consistency requires the clinical assistance and has to be treated accordingly.

Though CAD systems and procedures have gained increasing acceptance in the field of medicine, they do suffer a few difficulties in translating human color perception into objectives that is analyzed by a computer. This is developing at a slower pace. Also, one of the main parameters in the assessment of burn wounds as color, for which an image - acquisition system has to be highly accurate. In this regard, burn depth is being evaluated by using thermo graphic image terahertz pulsed imaging, polarization - sensitive optical coherence tomography, reflectance spectrometer and Laser Doppler flex measurements. As their names, these methods are known for its complexity and high cost, which is again a disadvantage. To overcome these problems a digital photographic camera is used as a replacement for image - acquisition system, [Jefferson-Brain et al. (2019)]. Even this served as a problem as in practical situations, the conditions for its illumination becomes uncontrollable. This issue was addressed as tele-dermatology and it was concluded that, with highly specialization camera and either requirement being met, this can be used as a replacement for the complex acquisition system.

\section{Literature Review}

Presently the research work carried out in analyzing the skin burnt images is very nominal, so here there is a lot of scope for the improvement in the research fields. The major research work carried out in the field of medical image diagnosis, burnt images classification are studies and the research gaps or the future research directions observed were present presented in this section. Out of which, the research contributions from Spain were significant in the bio medical image processing.

The various research works relevant to the theme of the proposed work are investigated and presented as follows [Hansen et al. (1997)]. Proposed a clinical evolution based on the burnt images using the Optical reflection technique. Next, extended the work based on the image diagnosis and detected the presence of the ulcers. This research work has helped in the detection of the ulcers in an early stage and helped the diagnosis team to cure the disease in an improved manner using the computer-based image processing. Further it is understood that the authors have used the technique of detecting the ulcers by using the color of the image and its processing techniques. They developed a methodology of reading the hypsographical images in a quantified manner and assessed the wound image and its information. [Thiran et al. (1996)] Proposed an algorithm for the early detection of cancer cell or tissues using the mathematical modelling of the tissues based on the microscopic image and further classified it based on being benign and malignant. The proposed mathematical model obtains the values from each cell and categorizes the tissues into four major groups in order to classify the images in an improved manner.

The bio-medical image processing engineers' group have made an extensive survey on the plastic surgery's by using telemedicine, [Serrano et al. (1998)]. The burnt image is captured in digital image techniques and thus the transmission of this communication in telemedicine helps in diagnosing in case of plastic surgery.

In this work the researcher designed an innovative clinical instrument for the evaluation of the image in case of burnt images, [Abubakar et al. (2019)]. This identifies the depth of the burnt image based on its features like colour, in two ways i.e., true colour and false colour. This is estimated purely based on the probability concepts applied on the burnt images.

In this work the researchers has estimated the depth of the burnt image by using the radioactive isotope techniques, where in the burnt images has to undergo this process in order to estimate the depth of the burnt image it needs the setup of the radioactive test bed to diagnose the burnt area, [Bennet et al. (1957)]. Expressed 
the methodology in classifying the depth in the burnt images by using the colour and its texture with the aid of the neuron networks and fuzzy logic. The colour information of the image is extracted by using the classifiers and clustering techniques using the vectored techniques. Further, classified the burnt images based on the image depth and its feature based on colour using the Fuzzy ATMAP neural networks by using the non-linear SVM, [Afromowitz et al. (1988), Ugail et al. (2019), Georgopoulos et al. (2019)].

Based on the above research contributions in the proposed research work for the classification of the burnt images an Enhanced SVM algorithm is used based on the mathematical function and its kernels. Here, the kernel plays a vital role in transforming the information to the required form. In this process a various form of kernels with the SVM are studied and the comparative study is carried out to assess the best performing algorithm for the classification of the burnt image in an effective manner. Out of the vital algorithm for SVM it is observed that the linear, nonlinear, polynomial based along with the radial basis function and sigmoid based SVM algorithm performs better to that of all the existing state of art techniques.

\section{Enhanced Support Vector Machine (ESVM) Algorithm for Burn Boundary Determination}

In this research work the prime focus is made on the implementation of the Burn detection system with the aid of the Enhanced Support Vector Machine (ESVM) Algorithm. With this, the detection of the burnt image is carried out successfully with two phases, firstly focused on the Majority Voting Scheme (MVS) with the aid of the Graphical user interfaces and secondly the ESVM with the image pre-processing, image contrasting, image energy and multi-dimensional scaling etc., the detailed discussions are discussed in the subsequent sections.

\subsection{Majority Voting Scheme}

The researchers have developed some of the Graphical User Interfaces (GUIs) which has to deal with a large set of data let us say approximately around 10000 regions these regions are termed as super pixels in which the burn images are segmented. It is observed from the studies carried out that the detection of an image and labelling it may take around 10 minutes in which the time taken is critical and to reduce the time the research work help a lot for the Clinicians. To improvise the currently using imaging techniques, a data set of images from the reputed hospitals and the patients is collected say 50 to 100 burn images.

In order to investigate the collected images, we have used a simple Photoshop using a personal computer or a laptop through this process we are able to figure out the burn area from the un-burnt area and this process is observed to be a time effective and it is taken from around 30 seconds to 1 minute. This analysis is carried out using a simple majority voting scheme (MVS). In this scheme we have evaluated say 4 to for clinical images and did the process for three to four iterations out of which if the pixels are matching more than half we estimated it as an super pixel and finally labelled it as an burn area else it is treated it as an un burnt area and this algorithm is discussed in detail as shown in MVS Algorithm.

Further the labelling of the burnt area is carried out by using the burn area and burn boundary detection based on the super pixels. The match found with the clinical analysis is around $97 \%$ match area through this we can be able to implement the labelling technique with an improved accuracy.

$$
\begin{aligned}
& \text { Collect the Clinical Images say } 3 \text { to } 4 \\
& \text { If } \quad \text { Match is found more than half of the pixels } \\
& \text { then } \quad \text { Declare it as an Burnt area } \\
& \text { else } \quad \text { Declare it as an un burnt area } \\
& \text { end }
\end{aligned}
$$

\subsection{Support Vector based Machine Learning (SVML) Architecture}

The research work carried out by the researchers is based on the Stable Neural Networks (SNN) and this is further based on the sub set classifiers which are trained with the help of some cross-validation Strategies. These techniques can be able to detect only the burnt area with lesser accuracy. The detailed identification process of burned image is shown in Fig 1. 


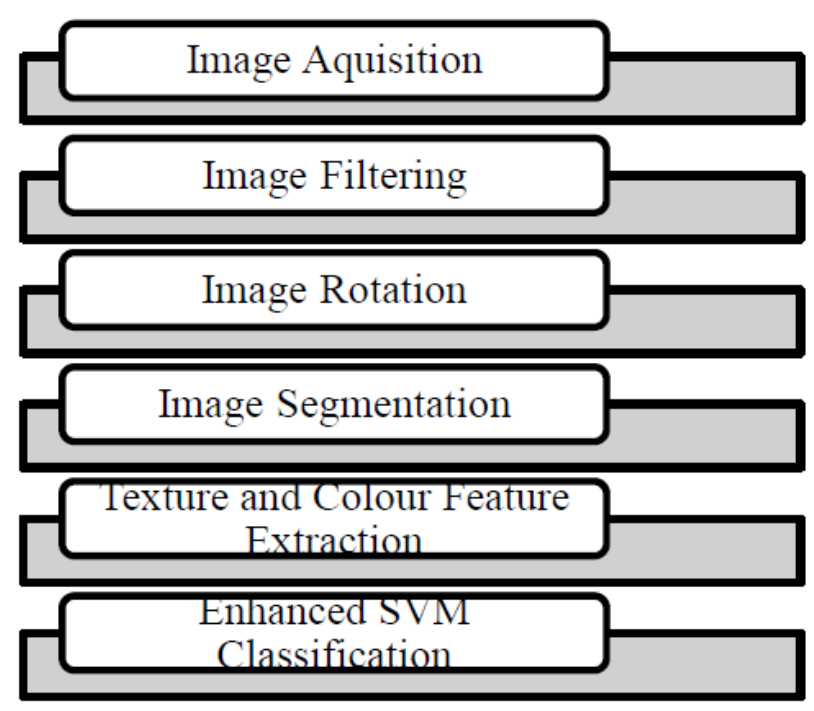

Fig. 1. The identification process of the Burnt Image

\subsection{Image Pre-Processing}

In order to de-noise the image used for the medical purpose from the segmented image by using the Euclidean distance. The de-noised image is referred as I and it is further rotated to make the necessary adjustment by an angle $\theta$ and the width and height are updated as follows in equations, Where $\mathrm{W}$ represents the Width, and $\mathrm{H}$ represents the Height of the original image.

$$
\begin{aligned}
& W_{\text {updated }}=2\left(\left.\right|_{2 \operatorname{Cos} \theta} ^{\underline{W}}|+|_{2 \operatorname{Sin} \theta}^{\underline{H}} \mid\right) \\
& H_{\text {updated }}=2\left(\left|\frac{H}{2 \cos \theta}\right|+\left|\frac{W}{2 \operatorname{Sin} \theta}\right|\right)
\end{aligned}
$$

In the above Equation the width and height are referred as the dimensions of the original image width and height respectively. Where Wupdated and Hupdated are the values of the respective updated width and height after the image is rotated by an angle $\theta$. Further the coordinated of the image (I) and the new image $I c$ are measured as follows with the Eq. (3) and (4).

$$
\begin{aligned}
& \left(a_{0}-a_{i 1}\right)=\left(a_{1}-a_{i 2}\right) \cos \theta-\left(b_{1}-b_{i 2}\right) \sin \theta \\
& \left(b_{0}-b_{i 1}\right)=\left(a_{1}-a_{i 2}\right) \sin \theta-\left(b_{1}-b_{i 2}\right) \cos \theta
\end{aligned}
$$

From the above equations the notations denoted $x 0$ and $y 0$ are the coordinates from the original image, where $a 1$ and $b 1$ are the coordinates of the transformed image; ai 1 and $b i$ are treated as the central coordinates used in the original image; ai2 and bi2 are the coordinated of the transformed image.

Usually for analyzing the sample a conventional medical diagnosis is performed in the laboratory environment for the bio medical signals, the image procession has taken a shape where we can analyze the burnt image and classify this based on analysis carried out for the images. The images processing techniques further helps us in extracting the features and this helps in the medical diagnosis and imaging using Fourier transform and 2D functions for the images in monochrome. Further the brightness of the images and the RGB colours profiles in the component is techniques are analyzed.

\subsection{Image Contrasting}

The Image Contrasting can be achieved by using the mathematical Equation Expressed in the Equation (5) as follows

$$
A_{I}=\sum_{i}^{L-1} \sum_{j}^{L-1}(i-j)^{2} G(i, j)
$$


Where the $|i-j|$ refers to the grey level difference between the pixels; where $G(i, j)$ refers to the Probability Distribution of the Grey Scale between the adjacent pixels; where $A I$ is the Contrast indicated and this parameter indicated the depth of the image grooves, As the contrast increases the Grooves are deeper and it is vice versa.

\subsection{Image Energy}

The Energy of the image is measures as per the Equation (6) as follows

$$
A_{E}=\sum_{i}^{L-1} \sum_{j}^{L-1}(G)^{2}(i, j)
$$

In order to find the texture of the image the Parameter $A E$ is used, the texture parameters of the energy model as achieved from the Grey scale model between the adjacent axis; where the $G(i, j)$ refers to the Probability Distribution of the Grey Scale.

\subsection{Method for Multi-dimensional Scaling}

Where the parameter I is used for number of images, and $I=25$ used in the experiment. The Matrix is $25 \times 25$.

$$
\Delta=\left[\begin{array}{rrr}
\delta_{11} & \delta_{12} & \ldots \ldots \ldots \delta_{1 I} \\
\delta_{21} & \delta_{22} & \ldots \ldots \ldots \delta_{2 I}
\end{array}\right]
$$

Where in the Matrix $\delta i, j$ where $i=1,2,3, I$ and $j=1,2,3, I$ and here the parameter I is not equal to $\mathrm{j}$. The Similarity matrix is built in with a variable as $\Delta$ as shown in Equation 7. This Matrix was obtained at the end of the comparison to find the similarity index.

\subsection{Scaling in Multidimensional Array}

The Similarity Matrix $\Delta$ is the input for the scaling in a multidimensional manner; this will help us in representing the data in an improvised interpretation. The $\Delta$ is a $I \times I$ Matrix, with an output of I Points, where each point in an object of a burnt image considering the configurations in geographical manner to produce the output in a spatial manner.

This process primarily estimates the Similarity between the points by using the distance in the map if the distance is high in the map which indicates the similarity of the objects is low.

In this scenario let us assume the points available in the dimensions are say $\mathrm{P}$ for which the points are $x 1$, $x 2, \ldots . x D$ the distance among them in a map can be represented as shown in the Eq. (7).

$$
\begin{aligned}
x_{1} & =\left(x_{11, \ldots . . .} x_{1 p, \ldots . .} x_{1 P}\right) \ldots \\
X_{I}=\left[x_{i}\right. & \left.=\left(x_{i 1, \ldots . .} x_{i p, \ldots . . .} x_{i P}\right) \ldots\right] . \\
x_{I} & =\left(x_{I 1, \ldots . . .} x_{I p, \ldots . .} x_{I P}\right)
\end{aligned}
$$

The Euclidian Distance between the desired points say $i$ and $j$, say for $i=1,2,3, \ldots 25$ and $j=1,2,3, \ldots .25$ is determined as shown in the Eq. (8) as follows

$$
\Delta=\left[\begin{array}{cccc}
d_{11} & d_{12} & \cdots & d_{1 I} \\
\vdots & & \ddots & \vdots
\end{array}\right] .
$$

By using this analysis the best suitable and the most suitable dimensions are estimated and form this maximum number of objects and this objects can be identified with a fixed number of objects in the input as follows as in Eq. (10).

$$
(I-1) \geq=4 P
$$

As per the above Equation the total numbers of Meaningful dimensions are given for $\mathrm{P}=4$.

\section{8. $k$ - Nearest Neighbour (kNN) Classifier}

The k- Nearest Neighbor Classifier $(\mathrm{kNN})$ is based on the voting based on the examining the nearest neighbor. The kNN algorithm identifies the $k$ objects in its group and sets the nearest sets. In this approach the selection of the neighborhood. This should not be too small or to too large rather it should be just right this can be understood with a simple analogy say for an instance to choose the nearest neighbor based on choosing the $k$ 
value. Where $x s$ and $y d$ are the $\mathrm{x}$ and $\mathrm{y}$ points of the scale and $d 2$ is the Euclidian distance between two point as expressed in Eq. (11).

$$
\text { Distance }\left(d^{2}\right)=(x s-y d)(x s-y d)
$$

\subsection{Proposed Enhanced Support Vector Machine (ESVM)}

The Support vector Machine (SVM) is basically a binary classifier used to classify the data based on the hyper plane of the data from one form to another form. It identifies the larger margins between the classes and classifies the best hyper plane for given classes.

The SVM is used in image processing for better image classification it has better accuracy by using the reliability prediction and also by reducing the redundant data the SVM is better than the query-based searching techniques.

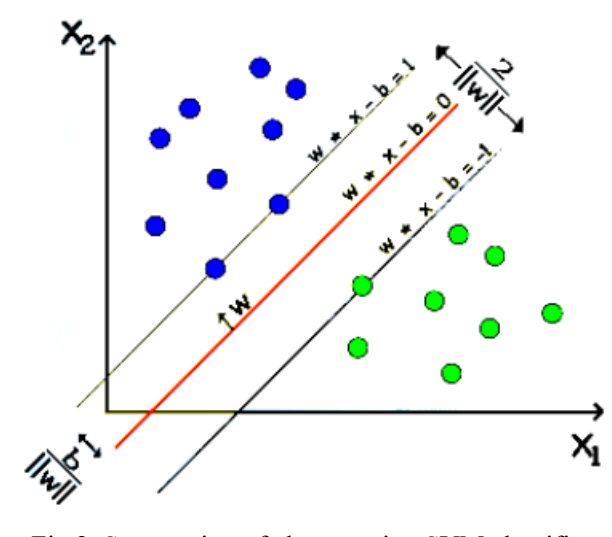

Fig.2. Segregation of classes using SVM classifier

The hyper-plane for $\langle w, x>+b=0$, where $W \in R d,<w, x>$ is the inner dot product of the $w$ and $b$ is real. As shown in figure 2 we have two support vectors indicated in green color and blue color in which each row indicates an observation and each column indicates a feature. The supervised kernel training is classified based on the supervised data in the group as data belonging to the vector in column with corresponding to each row and the corresponding row in the data.

In order to optimize the performance, the training parameters are changed dynamically for the performance optimization.

$$
\operatorname{class}(z)=\operatorname{sign}(<w, z>+b)
$$

For the given vector for a given class of $z$ the best optimal solution is as shown in Eq. (12).

$$
k(x, y)=(1+<x, y)^{d}
$$

The quadratic function for a dimension $d$ is given as in Eq. (13).

The image analysis of a burnt image is a vital aspect in grading it based on the machine learning approaches. The proposed research work is extended by upgrading the existing work with a Two-Stage Enhanced SVML Model based on Support Vector Machines (SVMs). The detailed discussion of the proposed model is discussed in step-by-step process and the procedure is discussed in detail as follows:

Step 1: Initially the data set if entirely trained to say $\mathrm{k}$ Subsets of equal magnitude using the SLIC algorithm in which the image dimensions are equally maintained in same size, in this process we have used and equal number of Super Pixels (SPs) for the trained images.

Step 2: the image of the burn area is captured and compared with the collected k-1 subsets the image processing tool box helps in analyzing the burn image and classify the burn and non-burn areas. Say $m$ burn super pixels are collected in which the $\mathrm{n}$ non burnt pixels are observed in which let us say that if $(n>m)$ form the total subsets collected from 1 st subset to k-1 subsets. In this manner if $m$ non burnt areas are selected form $\mathrm{n}$ burnt area we have $2 \mathrm{~m}$ number of training patterns. 
Step 3: The Cross-validation Scheme is used to train the SVM classifiers based on the $2 \mathrm{~m}$ patterns in training. In this process we split it into 10 to 12 Subsets of equal size folds out of the proposed subsets one will validate with the remaining training sets.

Step 4: The trained classifiers are used to classify the folds apart from the k-1subsets used in the training

Step 5: Use the trained classifier to classify regions in the subset other than k-1 subsets for the training.

Step 6: The instances in which if any incorrectly classified instances are found from above step 4 are sent to next stage

Step 7: The Steps from 2 to 5 are repeated k times until the subset is validated at least one time

Step 8: The Second Stage is progressed as in Step 3 using SVM binary Classifier.

As per the discussions carried out earlier in the previous sections, in the proposed technique an enhanced version of the Enhanced Support Vector Machine (ESVM) based method is proposed in order to improve the detection mechanism.

In spite of the improvements found with the proposed technique it also can be fine-tuned further in improving the accuracy as this has to be trained with improved set of training in case of burn image is complicated it becomes difficult in detecting the burn accurately if complete burn image is darn in visualization and computation due to this the image detection gets confused with the maximum -minimum based searching points in the turning methods for detection. This can be overcome by the aid of adjustments in the fusion regions and the bandwidths. In the detection of the burnt image processing the SVM perform betters with its own abilities. To improve still further Enhanced SVM based machine learning based approach is proposed by dealing the multidimensional features to convenience the protections in over fitting, the major idea behind the proposed work is about the non-linearly separable training with low feature space as well in the high dimension space, the mapping is carried out by using the feature vector based on the separate kernels for the low and high dimensional feature spaces.

The machine learning approach used in the image processing is divided into two phases firstly the training phase and the classifying phase, the proposed system uses system to use the Machine Learning (ML) algorithm to apprise the parameters used and helps in classifying the model. Further after collecting the burnt image the image is subjected to be standardized and segmented before the input is fed to the feature component. The training images are used for classifying the classes in the burnt images among number of classes.

\subsection{Training Model}

The experimental data projected as per ITU-R BT 500:10 standards in this standard each cell represents the pairing of the medical images from a scale of 0 to 10 as per the scale the image similarity is tested while the scale 0 indicates complete mismatch and 10 indicates completely.

Based on this training the data is estimated and the experimental data is presented for the physicians and further these results were presented in the similarity matrix. Further the rank of comparison is tested and trained to all the individual data.

The simulation begins with the establishment of the test with stabilization phase; this phase is just to make sure that everything is going correctly, with the help of the similarity index. Next the main experiment is initiated similar to the stabilization phase. Out of total 150 images we have compared 20 images we can see the pair of images as in Fig 3. And while presenting the pair a kind of randomness is followed. Initially the participant views the pairs of different degree or order this is to ensure that each individual performs a variety of testing, secondly the emplacement of the images further changes from the left side to the right side. 

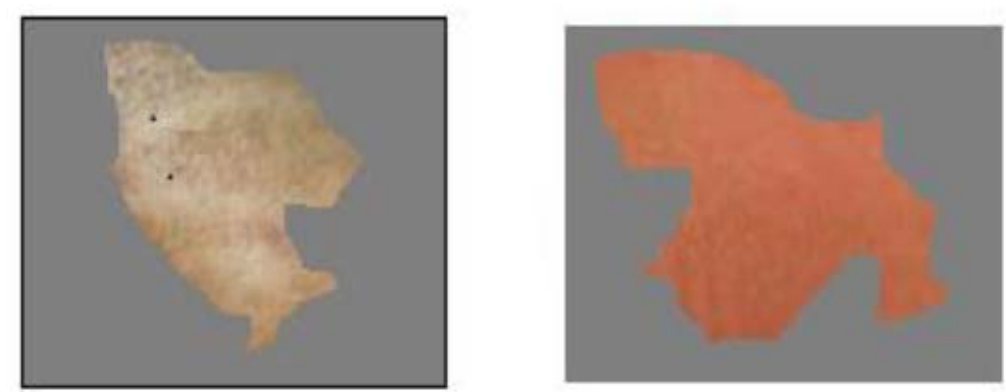

Fig. 3. An example of the pairs of images that physicians have to compare.

By using these methods, the observations are made consistently. The illumination of the images is compared with variant backgrounds in a grey compared with the burnt wound and with various luminous values until the better comparison is observed. Where, manual segmented image by the clinical experts can be seen in Fig 4 .
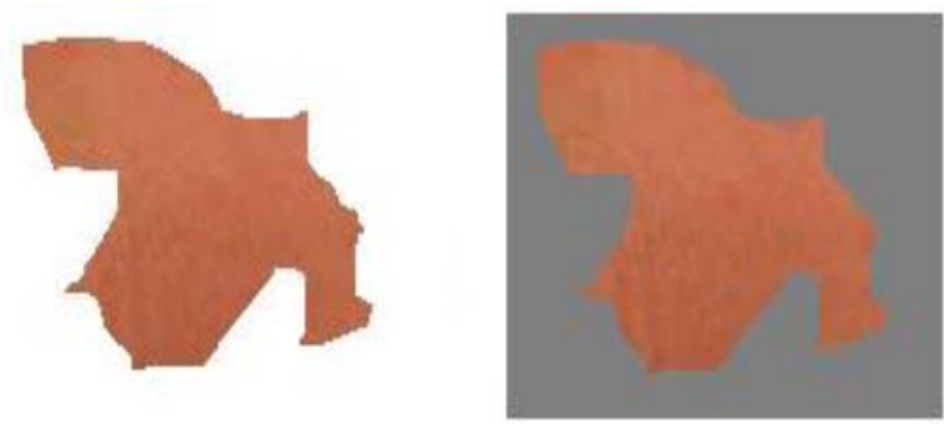

Fig. 4. Background was set to $\mathrm{L}=50$ (right image).

It is observed from the Fig 3 and 4 that if the back ground is used as white we feel more difficult in comparison of the images.

\section{Simulation Environment and Experimental Database}

The Acquisition of the skin burnt images is carried out by using an image capturing with the aid of a normal cameral used in mobile phones or the laptop-camera images in an user friendly manner, as these images may not in a position to assess the burnt image appropriately as these images may lack in isolation or lack of lightening, the images may have noise duo to its illumination or variation in its dynamic range with the variable settings in the lens and variable environmental conditions all these adjustments in the images needs to be carried out before assessing the image and the process involved in the proposed work is discussed in detail in the simulation environment section.

\subsection{Simulation Environment}

The simulation of the burnt image is carried out based on the data base or the data set in which we have assessed the images based on its classification for each set of classification we have taken 50 images for three grades it is a total of 150 images as shown in Table 1. The data base is built based on the images collected from various sources like web images, collected online, and finally of personal interested captured from individuals and volunteers from hospitals and those collected images are set to a jpeg format. 


\begin{tabular}{|l|l|l|}
\hline Name of the classifier & $\begin{array}{l}\text { Classification } \\
\text { time (in msec) }\end{array}$ & $\begin{array}{l}\text { No. of } \\
\text { classes }\end{array}$ \\
\hline Deep Learning (DL) & 4100 & 3 \\
\hline Random Forest (RF) & 9200 & 3 \\
\hline K Neighbour Neighbour (kNN) & 3700 & 3 \\
\hline Support Vector Machine (SVM) & 3250 & 3 \\
\hline Decision Tree (DT) & 3500 & 3 \\
\hline $\begin{array}{l}\text { Enhanced Support Vector Machine } \\
\text { (ESVM ) }\end{array}$ & 3650 & 3 \\
\hline
\end{tabular}

Table 1: Classification Time

\subsection{Experimental Database Training and Matching Methods}

An image data base is created for 150 burnt images with a variation of all the four degrees of the burnt images say burn degree I, II, III, IV respectively are given as Level 1 to level 4. In the analysis the main focus is made on Group II, III, and IV as the Group I Burns are of a minor concern it may also be caused due to the sun burn.in this scenario the range of images for degree II burns are 50 images and for degree III burns images are 50 and the degree IV burns are 50 images.

\begin{tabular}{|l|c|c|c|c|}
\hline Type & $\begin{array}{l}\text { Total } \\
\text { number } \\
\text { of } \\
\text { images }\end{array}$ & $\begin{array}{l}\text { Online/ } \\
\text { Web }\end{array}$ & $\begin{array}{l}\text { Real } \\
\text { Time } \\
\text { Images }\end{array}$ & $\begin{array}{l}\text { Through } \\
\text { printed } \\
\text { materials }\end{array}$ \\
\hline $\begin{array}{l}\text { I Grade } \\
\text { Burn } \\
\text { Degree }\end{array}$ & 50 & 9 & 39 & 2 \\
\hline $\begin{array}{l}\text { II Grade } \\
\text { Burn } \\
\text { Degree }\end{array}$ & 50 & 7 & 37 & 6 \\
\hline $\begin{array}{l}\text { III Grade } \\
\text { Burn } \\
\text { Degree }\end{array}$ & 50 & 6 & 36 & 8 \\
\hline
\end{tabular}

Table 2: Burnt image Data Base

The samples in case of higher degree are less as we encounter the minimal number of survival rage of this degree burns. Out of these images collected is divided to two equal half's for training and the testing purposes finally the data base is created for all the data sets in the system to perform the analysis. The final test folder used in training is as shown in Table 2.

\begin{tabular}{|l|c|c|c|c|}
\hline Type & $\begin{array}{l}\text { Total no. } \\
\text { of } \\
\text { Images }\end{array}$ & $\begin{array}{l}\text { Traine } \\
\mathrm{d} \\
\text { Images }\end{array}$ & $\begin{array}{l}\text { Improper } \\
\text { classificatio } \\
\text { n }\end{array}$ & $\begin{array}{l}\text { Efficie } \\
\text { ncy }\end{array}$ \\
\hline $\begin{array}{l}\text { I Grade } \\
\text { Burn } \\
\text { Degree }\end{array}$ & 50 & 37 & 13 & 74 \\
\hline $\begin{array}{l}\text { II Grade } \\
\text { Burn } \\
\text { Degree }\end{array}$ & 50 & 41 & 7 & 82 \\
\hline $\begin{array}{l}\text { III } \\
\text { Grade } \\
\text { Burn } \\
\text { Degree }\end{array}$ & 50 & 39 & 9 & 78 \\
\hline
\end{tabular}

Table 3: Training and Matching methods in Image classification 


\section{Experimental Results}

In this work the real time image classification is carried out using the machine learning approach to classify the degree of burns and to identify the tissue type and to assess the healing assessment. From experimental results carried out the Enhanced SVM classifications is more accurate. Further in order to assess the performance of the proposed system the precision, false acceptance and false rejection rates are investigated for various grades and presented.

The classification of the burnt image The precision, False Acceptance Rate (FAR) and False Reject Rate (FRR) is the common criterion to evaluate the classification rate, simplify given by the Equations (14) - (16) respectively

$$
\begin{aligned}
& \text { Precesion }=\text { correctly classified images/Total number of images } \\
& \text { False Acceptance Rate }(F A R)=(\text { False Classified images }) /(\text { classified images }) \\
& \text { False Rejection Rate }(F R R)=\text { False Rejected images / Rejected images }
\end{aligned}
$$

\begin{tabular}{|c|c|c|c|c|c|c|c|}
\hline 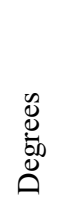 & 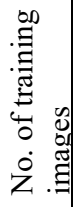 & 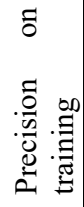 & 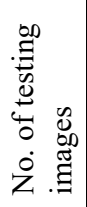 & 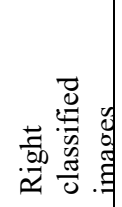 & $\begin{array}{l}\frac{0}{d} \\
.0 \\
\frac{0}{0} \\
\frac{0}{0} \\
0 \\
0\end{array}$ & 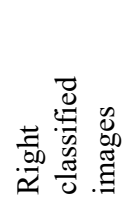 & 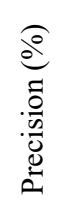 \\
\hline I & 50 & 100 & 37 & 13 & 74 & 68 & 78 \\
\hline II & 50 & 100 & 41 & 9 & 82 & 72 & 89 \\
\hline III & 50 & 100 & 39 & 11 & 78 & 6 & 86 \\
\hline
\end{tabular}

\begin{tabular}{|c|c|c|c|c|c|c|c|}
\hline \multirow[b]{2}{*}{ 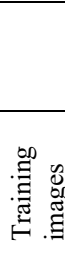 } & \multirow[b]{2}{*}{ 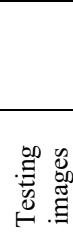 } & \multicolumn{3}{|c|}{$\begin{array}{l}\text { Right classified } \\
\text { images }\end{array}$} & \multicolumn{2}{|c|}{ Precision (\%) } & \multirow[b]{2}{*}{ 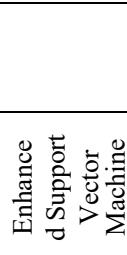 } \\
\hline & & 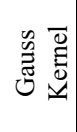 & 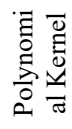 & 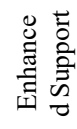 & 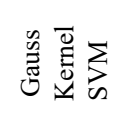 & 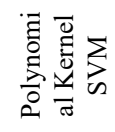 & \\
\hline 150 & 150 & 107 & 115 & 126 & $71.33 \%$ & $76.66 \%$ & $84.66 \%$ \\
\hline
\end{tabular}

Table 4: Burning Image Classification Results Using SVM

Table 5: Classification Results Using Gauss and Polynomial Kernel and ESVM

From the Eq. (14) (15) (16) the precision, False Acceptance Rate (FAR) and False Rejection Rate (FRR) for the degree II ,III, IV were classified and presented in Fig 5, it is observed that the variations in degree IV are more as the FAR and FRR is high and low respectively.

The image implant is too low in this kind of images the rejection rate is observed to be too low. Fig 6 shows the classification of the kNN, SVM and ESVM are compared with the burning, the ESVM has the improved rate of accuracy in the burnt image classification but the trade of between the trade of between the computational time to the accuracy. 


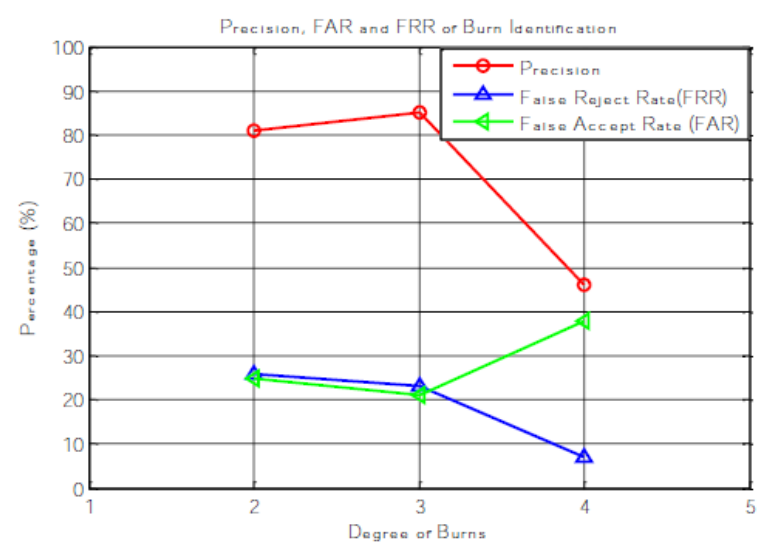

Fig. 5. Precision, FAR, FRR for Burnt image

The Experimental results are carried out for a fixed degrees of II,III,and IV of the burn level.

\section{Burning image classification of precision}

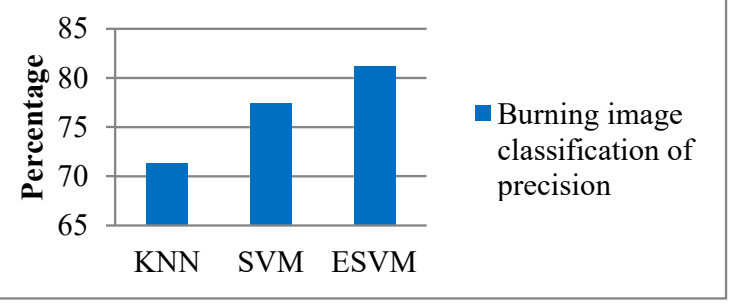

Fig. 6. Classification of Burnt images with precision.

Fig 7 shows the proposed work in assessing the healing the chronic wounds and the burnt images. the burnt image changes its phases from necrotic eschar to the slough and then to the granulation to the healing tissues.

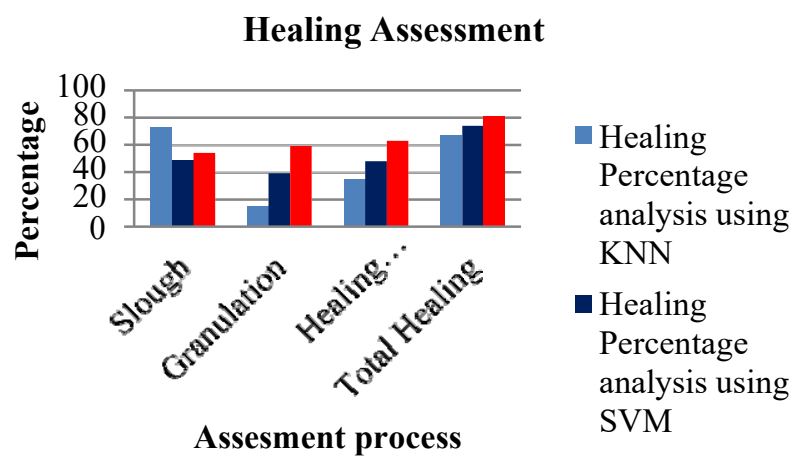

Fig 7. Healing Assessment process analysis.

The tissues of the skin cells are transformed into a healing the epithelial tissues as the burnt /wound grows and the edges are closed naturally. 


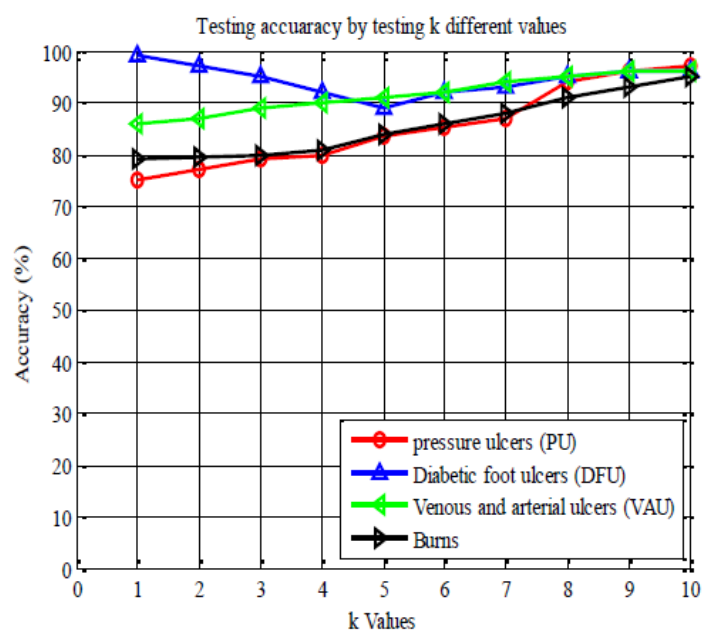

Fig 8. Testing and validation of Accuracy for various $\mathrm{k}$ values.

Fig 8 Shows the $k=1,2, \ldots 10$ to achieve the better performance, where the $k$ represents the elements of the number of feature vectors. In this we found that $k=9$ and $k=10$ give the best results for the assessment. In the figure it is shown that the accuracy of the system with respect to the different values is assessed based on the $k$ parameters for different burnt/ wound images of the skin tissues.

Further all the obtained results are cross validated and the proposed system accuracy is achieved as $96.5 \%$, and on the other hand the DT, kNN, SVM obtained $91.2 \%, 91.9 \%$ and $95.1 \%$ respectively. The proposed system achieves and average accuracy of $96.5 \%$ of all types of the burnt images of all degrees. Thus, the proposed system outperforms the state of are techniques with a $k=9$-fold cross validation. Further this system can easily distinguish the burnt images and its tissues in curing the skin tissues.

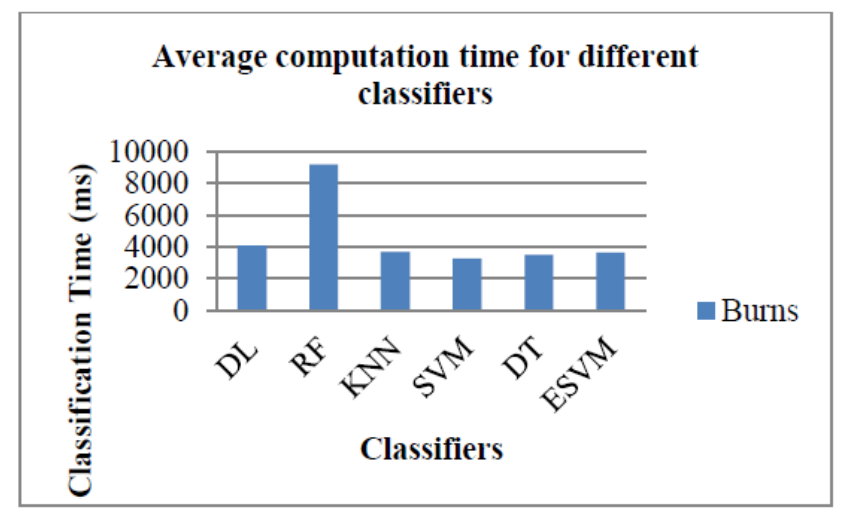

Fig. 9. Classification time of various classifiers

Figu 9 The accuracy of the overall system is assessed and then it is compared with the different classifiers say Decision Tree (DT), k- Nearest Neighbor (kNN), Support Vector Machine (SVM), Enhanced Support Vector Machine (ESVM), and Random Forest (RF).

\begin{tabular}{|l|r|}
\hline \multicolumn{2}{|c|}{ Success Rate } \\
\hline Classification in burn depths using kNN & $71.3 \%$ \\
\hline Classification in burn depths using SVM & $77.5 \%$ \\
\hline Classification in burn depths using ESVM & $81.2 \%$ \\
\hline
\end{tabular}

Table 4: Classification Success Rate

To assess the image data basses, a total of 150 images are collected. The kNN classifier and SVM along with ESVM are used to calculate the success rate of all the burnt images. For each segment for 3 degree 50 images for each are allocated and the experimental set up is trained and the burnt image depth is classified among the training images.

\section{Conclusion}

The assessment of the burnt image classifications in the image processing play a major role in the identification of the severity of burnt images, further this helps in assessing the proper treatment. The burnt images have the 
combinations of the Red, Green, and Blue colours. The burnt images spread to the inner layers in its nature, the RGB have certain patterns with certain probability distributions. The precision analysis helps the doctors in treating the burnt inner layers of the skin. The research work has proposed the classification of burnt image to out in the work applies two stages enhanced SVM training to classify the burnt images. The proposed techniques classify the degrees of the burnt images in ESVM.in which the classification is carried out in training phase and classification phase and the precision study and False Accept Rate (FAR) and False Reject Rate (FRR) is analyzed. The precision rate in ESVM is $84.6 \%$. And the success rate is achieved as $82.9 \%$. the burnt image accuracy is observed as $93 \%$ and classification time for ESVM is a bit higher than SVM in spite the accuracy is improved corporately and all the state of art classifiers are taken into account for the classification time for Deep Learning (DL), Random Forest (RF), k-Nearest Neighbor (kNN), Support Vector Machine(SVM), Decision Tree (DT) Enhanced Support Vector machine (ESVM) are analyzed and the ESVM performs better when the trade of between the accuracy and classification time is considered. The ESVM uses the Two- stage training and classification to improve the accuracy and outperforms with state-of art classifier techniques.

\section{References}

[1] Abubakar, A.; Ugail, H.; Bukar, A. M.; Aminu A. A.; Musa, A. (2019): Transfer Learning Based Histopathologic Image Classification for Burns Recognition, International Conference on Electronics, Computer and Computation (ICECCO), pp. 1-6.

[2] Acha, B.; Serrano, C.; Fondón, I.; Gómez-Cía, Y. (2013): Burn Depth Analysis Using Multidimensional Scaling Applied to Psychophysical Experiment Data, IEEE Transactions on Medical Imaging, 32(6), pp. 1111- 1120.

[3] Afromowitz, M. A.; Callis, J. B.; Heimbach, D. M.; DeSoto, L. A.; Norton, M. K. (1988): Multispectral imaging of burn wounds: a new clinical instrument for evaluating burn depth, IEEE Transactions on Biomedical Engineering, 35(10), pp. 842-850.

[4] Bennett, J. E.; Kingman, R. O. (1957): Evaluation of Burn Depth by the use of Radioactive Isotope- by An experimental study, Plolic and reconstructive surgery, 20(4), pp. 261-272.

[5] Dahel, S. K.; Xiao, Q. (2003): Accuracy performance analysis of multimodal biometrics, IEEE Systems, Man and Cybernetics Society Information Assurance Workshop, pp. 170-173.

[6] Dai, Y.; Liu, W.; Li, H.; Liu, L. (2020): Efficient Foreign Object Detection Between PSDs and Metro Doors via Deep Neural Networks, IEEE Access, vol. 8, pp. 46723-46734.

[7] Georgopoulos, N.; Stavrakoudis, D.; Gitas, I. Z. (2019): Object-Based Burned Area Mapping Using Sentinel-2 Imagery and Supervised Learning Guided by Empirical Rules, IEEE International Geoscience and Remote Sensing Symposium, pp. 9980-9983.

[8] Hansen, G. L.; Sparrow, E. M.; Kokate, J. Y.; Leland, K. J.; Iaizzo, P. A. (1997): Wound status evaluation using color image processing, IEEE Transactions on Medical Imaging, 16(1), pp. 78-86.

[9] Jefferson-Brain, T. L.;Coupe, A. D.; Burns, M. D.; Clarkson, W. A.; Shardlow, P. (2019): Alignment of Higher-Order Mode SolidState Laser Systems with Machine Learning Diagnostic Assistance, Conference on Lasers and Electro-Optics Europe \& European Quantum Electronics Conference.

[10] Khalil, A.; Elmogy, M.; Ghazal, M.; Burns, C.; El-Baz, A. (2019): Chronic Wound Healing Assessment System Based on Different Features Modalities and Non-Negative Matrix Factorization (NMF) Feature Reduction, IEEE Access, vol. 7, pp. 80110-80121.

[11] Serrano, C.; Rao, L.M.; Acha, B. (1998): Evaluation of a Telemedicine Platform in a burn Unit. Proc. IEE Int. Conf. on Information Technology Applications in Biomedicine, pp.121- 126.

[12] Thiran, J. P.; Macq, B. (1996): Morphological feature extraction for the classification of digital images of cancerous tissues. IEEE Trans Biomed Eng. 43(10), pp. $1011-1020$.

[13] Ugail. H. et al. (2019): A Deep Learning Approach to Tumour Identification in Fresh Frozen Tissues, International Conference on Software, Knowledge, Information Management and Applications (SKIMA), pp. 1-6.

[14] Yadav, D. P.; Sharma, A.; Singh, M; Goyal, A. (2019): Feature Extraction Based Machine Learning for Human Burn Diagnosis from Burn Images, IEEE J. of Translational Engineering in Health and Medicine, vol. 7, pp. 1-7.

[15] Wang, L.; Pedersen, P. C.; Agu, E.; Strong, D. M.; Tulu, B. (2017): Area Determination of Diabetic Foot Ulcer Images Using a Cascaded Two- Stage SVM-Based Classification, IEEE Transactions on Biomedical Engineering, 64(9), pp. 2098-2109.

[16] ZHAO, H et al. (2019): Fault diagnosis of wind turbine bearing based on stochastic subspace identification and multi-kernel support vector machine. J. Mod. Power Syst. Clean Energy 7, pp. 350-356.

\section{Authors Profile}

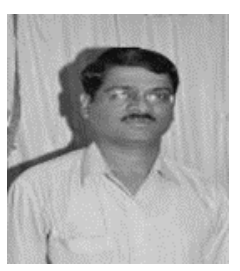

Somashekhar G. C. graduated from Karnataka university in Electronics and Communication. Engineering and MTech from S.K. University in Communications and Signal Processing. He is pursuing Ph.D. from VTU Belagavi and currently working as associate professor in Rajiv Gandhi Institute of Technology, Bangalore in the Department of Electronics and Communication Engineering. His area of interest is image processing.

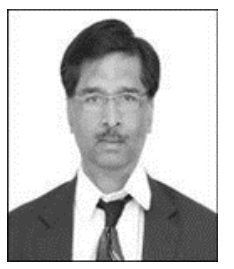

H. B. Phani Raju graduated from Bangalore University. He did his M.E. in power systems from UVCE, Bangalore University and $\mathrm{PhD}$ in Reliability Analysis Using Artificial Intelligence from VTU, Belagavi. He has published several research papers in International Journals and Conferences. At present, he is serving as vice principal at Sridevi Institute of Technology, Tumkur. 